\title{
Frauen in der Informatik: Können sie mehr als sie denken? Eine Analyse geschlechtsspezifischer Erfolgserwartungen unter Informatikstudierenden ${ }^{1}$
}

\begin{abstract}
Zusammenfassung
Obwohl ein Anstieg des Frauenanteils in den Informatikstudiengängen zu verzeichnen ist, gilt die IT-Branche nach wie vor als Männerdomäne. Der weibliche Anteil in deutschen IT-Abteilungen beträgt knapp 10 Prozent (Weitzel et al. 2017). Ein Grund für die mangelnde Präsenz der Frauen im IT-Bereich könnte die geringere Erfolgserwartung der Studentinnen im Studium sein. In diesem Beitrag wird untersucht, ob vorangegangene Schulleistungen sowie intrinsische Motivation für die Studiengangswahl, nämlich Begabung und Interesse für das Fach, die subjektive Einschätzung des Studienerfolgs von Informatikstudierenden beeinflussen. Obwohl Studentinnen sich im Vergleich zu ihren Kommilitonen in ihren durchschnittlichen Mathematikleistungen nicht signifikant unterscheiden und sie im Durchschnitt die bessere Abiturabschlussnote erzielen, unterschätzen sie sich in ihrem persönlichen Studienerfolg signifikant, insbesondere in stark techniklastigen Informatikstudiengängen. Ebenso können Studentinnen von einer hohen intrinsischen Motivation, hinsichtlich ihrer Erfolgseinschätzungen im Studium nicht profitieren. Die durchgeführte Analyse bezieht sich auf das Datenmaterial aus dem ESF-Forschungsprojekt "Alumnae Tracking".
\end{abstract}

\section{Schlüsselwörter}

Geschlecht, Informatik, Studienerfolg, Schulische Leistungen, Intrinsische Motivation

\author{
Summary \\ Women in computer science: Are they more \\ capable than they think? An analysis of gen- \\ der-specific expectations of success among \\ computer science students
}

The IT industry is still regarded as being male dominated. In Germany, the share of women in IT departments is just under 10\% (Weitzel et al. 2017). One reason for the lack of female professionals in this field could be female computer science students' lower expectations of success. This article examines whether previous school performance and intrinsic motivation, especially a talent for and interest in the subject (which were important when choosing a degree course), influence computer science students' assessments of academic success. The analysis shows that average final grades in Mathematics do not differ statistically significantly between male and female students. In fact, the study shows that female students achieve a significantly higher average final grade in high school. Nevertheless, female students, particularly in very technical computer science courses, significantly underestimate their academic success. Likewise, female students are unable to rate their academic success positively, even when their motivation is intrinsically high. The data presented here were taken from the ESF research project "Alumnae Tracking".

\section{Keywords}

gender, computer science, academic success, school performance, intrinsic motivation

1 Wir bedanken uns herzlich bei Prof. Dr. Sandra Buchholz und Dr. Anja Gärtig-Daugs für wertvolle Diskussionen und bei Susanne Gall, Verena Pfeiffer und Sophie Vollmar für ihre Unterstützung bei der Datenerhebung und -aufbereitung. 


\section{Einleitung}

Das Fehlen von Fachkräften in der Informatik wird seit mehreren Jahren sowohl in der Politik als auch in der Wirtschaft diskutiert (Bundesregierung 2013; Bundesverband Informationswirtschaft Telekommunikation und neue Medien e. V. 2014). Trotz intensiver Bemühungen haben bildungs-, hochschul- und wirtschaftspolitische Fördermaßnahmen zur Kompensation des Nachwuchsmangels in der Informatik bisher nicht zum gewünschten Erfolg geführt (Bundesministerium für Bildung und Forschung 2009). Ein besonderes Ziel war es dabei auch, dem Nachwuchsmangel durch eine Erhöhung des Frauenanteils in IT-Berufen und Informatikstudiengängen zu begegnen (Bundesministerium für Bildung und Forschung 2009). Bereits frühere sowie neuere Ergebnisse deuten darauf hin, dass Frauen im IT-Bereich unterrepräsentiert sind (Kempf 2012; Weitzel et al. 2017). Der bundesweite Anteil der Studentinnen in Informatikstudiengängen ist in den vergangenen Jahren zwar gestiegen: 1997 waren 18,5\% der Studierenden in Informatikstudiengängen weiblich; im Wintersemester 2015/2016 waren es knapp 25\% (Kompetenzzentrum Technik - Diversity - Chancengleichheit 2016). Allerdings sind Studentinnen häufiger in interdisziplinär ausgerichteten als in reinen Informatikstudiengängen anzutreffen (Kompetenzzentrum Technik - Diversity - Chancengleichheit 2014). Eine zentrale Ursache für die geringere Beteiligung von Frauen in der Informatik wird darin gesehen, dass die Informatik noch immer als Männerdomäne wahrgenommen wird (Schneider/Stenke 2016) und weibliche Vorbilder fehlen (Kompetenzzentrum Technik - Diversity - Chancengleichheit 2014). Hinzu kommt, dass junge Frauen ihre Eignung für den MINT-Bereich systematisch unterschätzen (Struwe 2017). So zeigt eine Studie der TU München, dass $80 \%$ der befragten jungen Erwachsenen sich darüber einig waren, dass IT ein Berufsfeld für Männer und nicht für Frauen ist (Broy/Denert/ Engeser 2008). Dass es berufsspezifische Geschlechterstereotype gibt, ist bekannt und wurde in verschiedenen Studien dokumentiert (Deutscher Gewerkschaftsbund 2014; Middendorff et al. 2013; Hachmeister/Harde/Langer 2007). Während junge Frauen Studiengängen der Sprach- und Kulturwissenschaften den Vorrang geben, favorisieren junge Männer den MINT-Bereich (Kompetenzzentrum Technik - Diversity - Chancengleichheit 2015; Middendorff et al. 2013).

Dieser Beitrag setzt an dem Punkt an, an dem sich junge Männer und Frauen bereits für ein Informatikstudium entschieden haben. Ziel der vorliegenden empirischen Studie ist es, zu untersuchen, ob Studentinnen und Studenten ihre Chancen, das Informatikstudium erfolgreich abzuschließen, unterschiedlich wahrnehmen. Insbesondere geht es darum, zu verstehen, inwiefern geschlechtsspezifische Unterschiede im erwarteten Studienerfolg dadurch zu erklären sind, dass Studierende unterschiedliche Fähigkeiten (Abitur- und Mathematikleistungen) mitbringen und in der Studienwahl von einer unterschiedlichen intrinsischen Motivation ${ }^{2}$ (Interesse und Begabung für das Fach) geleitet werden.

Die Frage ist hier: Unterschätzen sich Studentinnen stärker in techniklastigen Informatikstudiengängen wie z. B. der Angewandten Informatik?

2 In Anlehnung an Publikationen des HIS (Heine et al. 2005; Hachmeister et al. 2007) wird auch hier intrinsische Motivation mit Interesse und Begabung für das Fach operationalisiert. 
Es liegen bereits vielfältige Befunde zur geschlechtsspezifischen Selbsteinschätzung sowie zu Erfolgserwartungen vor (Kling et al. 1999; Skaalvik/Skaalvik 2004; Kessels 2012; Skorepa/Greimel-Fuhrmann 2009; Hannover 2007; Zimmer/Burba/ Rost 2004; Klieme/Neubrand/Lüdtke 2000; Hannover/Bettge 1993; Heatherington et al. 1993). Nach unseren Recherchen fehlt bislang jedoch eine explizite Untersuchung für den Bereich Informatik, vor allem, da entsprechende Daten bisher nicht vorlagen, die eine solche Analyse ermöglicht hätten. In der vorliegenden empirischen Untersuchung wird deshalb auf neu verfügbare Daten aus dem Forschungsprojekt „Alumnae Tracking $^{\text {“3 }}$ zurückgegriffen. In diesem Projekt wurden Studierende verschiedener Informatikstudiengänge der Fakultät Wirtschaftsinformatik und Angewandte Informatik (WIAI) ${ }^{4}$ an der Universität Bamberg u. a. zu objektiven Merkmalen (wie z. B. Schulnoten) und subjektiven Merkmalen (wie Erfolgserwartungen im Studium und motivationalen Aspekten bei der Studienwahl) befragt.

Der Beitrag ist wie folgt aufgebaut: Im ersten Schritt findet eine theoretische Auseinandersetzung mit dem Forschungsgegenstand und bisherigen Befunden statt. Daraus werden die Forschungshypothesen entwickelt. Daran anschließend wird das Forschungsdesign, d.h. die genutzten Daten, Methoden und Operationalisierungen, vorgestellt. Diesem Teil folgen die Ergebnisse der empirischen Analysen. Der Beitrag endet mit einer kurzen Zusammenfassung und Diskussion der Erkenntnisse.

\section{Theoretischer Hintergrund und bisherige Erkenntnisse}

In diesem Beitrag geht es darum, zu untersuchen, ob es geschlechtsspezifische Unterschiede in der persönlichen Einschätzung des Studienerfolgs gibt und ob schulische Leistungen sowie Interesse und Begabung für das Fach Informatik geschlechtsspezifische Einschätzungen beeinflussen. ${ }^{5}$ Auf der Grundlage des theoretischen Konzepts von Deaux und LaFrance (1998), die in ihrem theoretischen Modell die Bedeutung von Geschlechterrollen betonen, soll der Zusammenhang der geschlechtsspezifischen Selbsteinschätzung und der eigenen Leistungsfähigkeit diskutiert werden. Dazu ist es notwendig, in einem weiteren Schritt die Entwicklung des akademischen Selbstkonzepts mithilfe des „Internal/External Frame of Reference“-Modells nach Marsh (1986) näher zu beleuchten.

Deaux und LaFrance (1998) gehen in ihrem theoretischen Ansatz davon aus, dass Geschlecht eine bedeutende Rolle bei aktiven Wahrnehmungs-, Erwartungs- und Bewertungsprozessen spielt. Flexibles geschlechtstypisches Verhalten in einer aktuellen Situation wird von der Selbst- und Fremdwahrnehmung bestimmt (Deaux/LaFrance

3 Alumnae Tracking: https://www.uni-bamberg.de/wiai/gremien/frauenbeauftragte/alumnaetracking/ (Zugriff: 03.11.2017). Gefördert wurde das Projekt vom Europäischen Sozialfonds (ESF), dem Bayerischen Staatsministerium für Arbeit und Sozialordnung, Familie und Integration (vormals Bayerisches Staatsministerium für Arbeit und Sozialordnung, Familie und Frauen) sowie der Technologie-Allianz Oberfranken (TAO).

4 Die Fakultät WIAI wurde erst 2001 errichtet. Sie besitzt einen interdisziplinären Zuschnitt, der die auf den Wirtschaftswissenschaften und der Informatik aufbauende Wirtschaftsinformatik mit der kultur- und humanwissenschaftlich ausgerichteten Angewandten Informatik verbindet. Ebenso existieren klassische Fachgebiete der Theoretischen und Praktischen Informatik.

5 Der ausgewählte Literaturüberblick stellt nicht den Anspruch auf Vollständigkeit, sondern dient der Herausbildung überprüfbarer Hypothesen. 